\title{
Acute Myocardial Infarction with Microthrombi in Cardiac Small Vessels after COVID-19 Vaccination (ChAdOx1 nCov-19): A Case Report
}

Sohyung Park, Byung-Ha Choi

Department of Forensic Investigation, National Forensic Service, Wonju, Korea

Received: October 29, 2021

Revised: November 15, 2021

Accepted: November 23, 2021

\section{Correspondence to}

Sohyung Park

Department of Forensic Medicine, National Forensic Service, 139

Jiyang-ro, Yangcheon-gu, Seoul 08036, Korea

Tel: +82-2-2600-4614

Fax: +82-2-2600-4828

E-mail:zzeva@korea.kr
We present the postmortem findings of an unexpected death due to acute myocardial infarction (AMI) with microthrombi and thrombosis in other vessels after the first dose of coronavirus disease 2019 (COVID-19) vaccination (ChAdOx1 nCov-19). The deceased was a 69-year-old woman who complained of nonspecific symptoms shortly after vaccination and was found dead on the sixth day. Postmortem examination revealed AMI and complications (left ventricular rupture, hemopericardium) with microthrombi in small cardiac vessels, which are similar to the characteristic findings of myocardial injury caused by microthrombi in patients with COVID-19. Nonobstructive thromboembolism in the pulmonary arteries and inferior vena cava, and fibrin microthrombi in some small vessels in the brain were also observed. It is unclear whether these findings are associated with COVID-19 vaccination, and further comprehensive studies are needed.

Key Words: COVID-19 vaccines; Thrombosis; Myocardial infarctions

\section{Introduction}

Since the coronavirus disease 2019 (COVID-19) vaccine (ChAdOx1 nCov-19) was introduced in South Korea, various adverse events have been reported in healthcare workers, but most of them were not fatal [1]. The incidence of severe adverse events, including encephalopathy, Guillain-Barre syndrome, thrombocytopenic purpura, and anaphylaxis, has been reported to be $0.03 \%$ [1]. However, we recently experienced an unexpected fatal case after COVID-19 vaccination (ChAdOx1 nCov-19) that cannot be included in such known adverse events after vaccination. Hence, we present the postmortem findings of this case with a review of the literature.

\section{Case Report}

The deceased was a 69-year-old woman who was found unresponsive kneeling down before the toilet in the restroom in the early morning. The deceased received the first dose of COVID-19 vaccination (ChAdOx1 nCov19) 6 days prior to her death. The deceased complained of nausea on the day of vaccination and then some pain in the vaccinated site of the arm for the next 3 days. On the fifth day after vaccination, she felt herself 
unwell with general weakness and back pain. On the sixth day, the deceased was found dead by her daughter. Cardiopulmonary resuscitation was not performed. The deceased had medical history of uterine cervical cancer (cured), diabetes mellitus, hypothyroidism, and osteoporosis. The deceased was consulted to our institute for postmortem examination because of her unexpected death after vaccination and the anticipated medicolegal issues.

On external examination, the deceased appeared well-nourished and clean. The body mass index was $26.8 \mathrm{~kg} / \mathrm{m}^{2}$ (height, $160 \mathrm{~cm}$; weight, $68 \mathrm{~kg}$ ). The injection site for vaccination was not clearly detected on the left arm. No significant injury was identified and otherwise unremarkable.

On internal examination, hemopericardium (mostly blood clots, approximately $400 \mathrm{~mL}$ ) was identified in the pericardium (Fig. 1A). Epicardial hemorrhage was observed at the apex of the left ventricle, and the blood permeated from the apex (Fig. 1B). The heart weighed $408 \mathrm{~g}$. Mild cardiomegaly was observed. The circumferences of the tricuspid, pulmonary, mitral and aortic valves were $12.0,8.5,10.0$, and $7.5 \mathrm{~cm}$, respectively. The coronary arteries were unremarkable. The myocardium and epicardium of apex revealed multifocal hemorrhages related to focal rupture (Fig. 1C). However, the areas of myocardial necrosis were not evident, and explicit transmural perforation sites could not be grossly recognized. The thicknesses of the left ventricular wall, septum, and right ventricular wall were $1.0,1.0$, and $0.4 \mathrm{~cm}$, respectively. The right and left pulmonary arteries and inferior vena cava revealed nonobstructive thromboemboli (Fig. 1D). No more thrombosis was identified in other major arteries and veins including the cerebral veins, splanchnic veins, and deep veins of the leg. The thyroid was not identified. The other internal organs were unremarkable. The subcutaneous and muscular layers of the vaccinated site on the left upper arm were grossly unremarkable.

Microscopic examination revealed multifocal (not confluent) myocardial necrosis with focal rupture, hemorrhage, and acute inflammation (Fig. 2A, B). The small arteries around the areas of myocardial necrosis revealed nonobstructive and eccentric fibrin microthrombi (Fig. 2C). Fibrin microthrombi were also identified in some small vessels in the frontal lobe of the brain (Fig. 2D). A thrombus was identified on the endocardium of the left ventricle. Acute thrombi were identified in the right and left pulmonary arteries and inferior vena cava (Fig. 2E, F). The other organs were unremarkable.

In the postmortem toxicological tests, a statin drug and acetaminophen were found to be positive within the therapeutic levels. The glycosylated hemoglobin level in the postmortem blood was also increased (7.1\%; reference range $<5.6 \%$ ) which is consistent with the history of diabetes mellitus. In other postmortem ancillary tests including blood alcohol level, ketone bodies, biochemistry, C-reactive protein, and thyroid function tests, the results were unremarkable. The anti-heparin/platelet factor 4 (PF4) antibody test was attempted by sending the sample to the outside hospital; however, the test could not be performed because of postmortem changes in the blood sample.

The cause of death was determined as acute myocardial infarction and acute complications (focal rupture of the left ventricle and hemopericardium) in a woman with thrombosis.

\section{Discussion}

This case illustrates an unexpected sudden cardiac death occurring on the sixth day after COVID-19 vaccination (ChAdOx1 nCov-19), an unusual pattern of focal myocardial infarction with focal rupture of the left ventricle, which was attributed to microthrombi in small vessels in the myocardium, without any epicardial coronary artery disease. In addition, acute nonobstructive thromboemboli in the right and left pulmonary arteries and inferior vena cava, and fibrin microthrombi in some small vessels of the cerebrum were observed. There was no medical history of cardiovascular diseases, venous thrombosis, a recent trauma, or recent surgery. The deceased did not have any disability in her daily life and was not bedridden.

Microthrombi in myocardial microvessels and focal myocardial necrosis in this case are unusual, compared with the usual features of typical cases of acute coronary syndrome, usually transmural myocardial infarction caused by epicardial coronary atherosclerosis 
and thrombosis. Microthrombi are microemboli chipped away from the epicardial coronary artery thrombosis and eroded plaque, which was proven by autopsy and clinicopathologic studies [2-4]. Focal myocardial necrosis is caused by microvascular obstruction due to microemboli [4]. In this case, focal myocardial necrosis with focal rupture was observed without any epicardial coronary artery disease. No culprit lesions including atherosclerotic plaque or thrombosis were identified in the epicardial coronary arteries. There was no history of chest pain, angina or other heart diseases.

Myocardial necrosis with microthrombi in cardiac small vessels in this case was similar to the cardiac findings in patients with COVID-19. Microthrombi led to focal myocardial necrosis occupying an area of $<1 \mathrm{~cm}^{2}$, which is different from the usual transmural myocardial infarction caused by epicardial coronary artery thrombi [5]. Nonocclusive and eccentric fibrin microthrombi were also identified microscopically in some patients with COVID-19 and without any ischemic myocardial injury [6]. Similarly in this case, multifocal myocardial necrosis (not confluent) was observed, which was not grossly evident. Nonocclusive and eccentric microthrombi were also observed in the small vessels in the myocardium
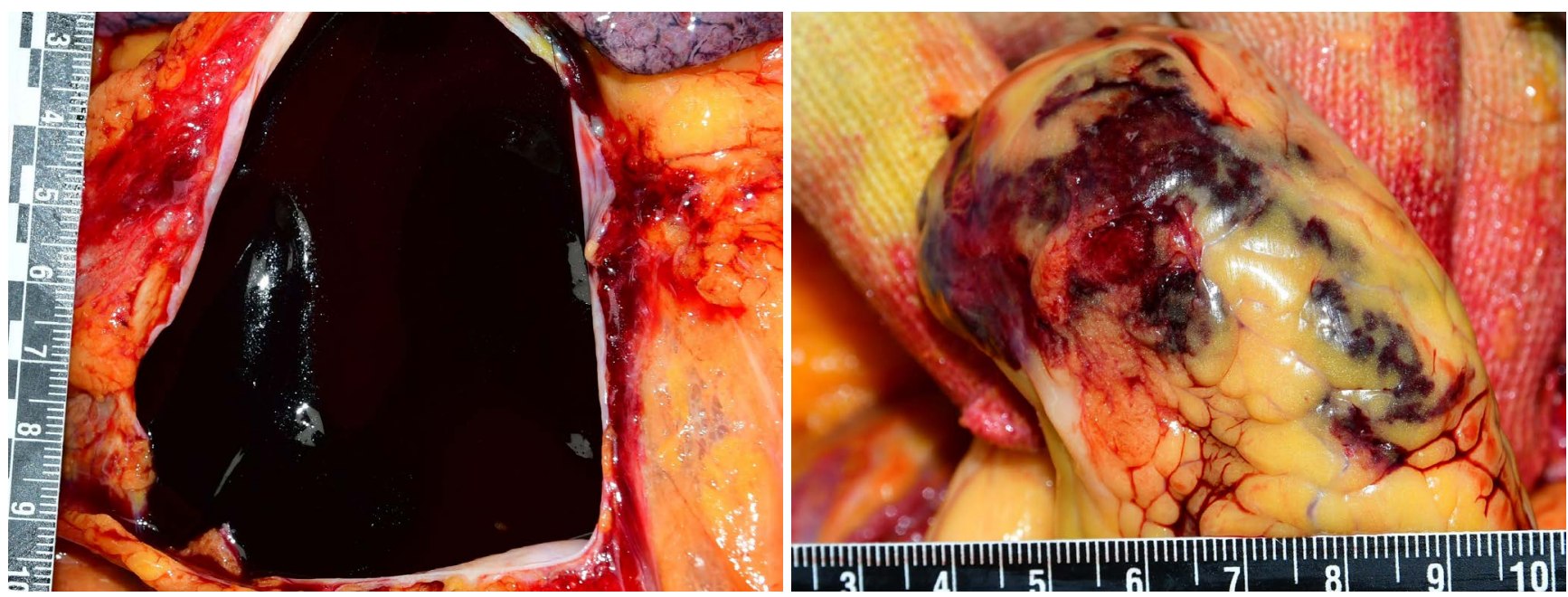

A

B
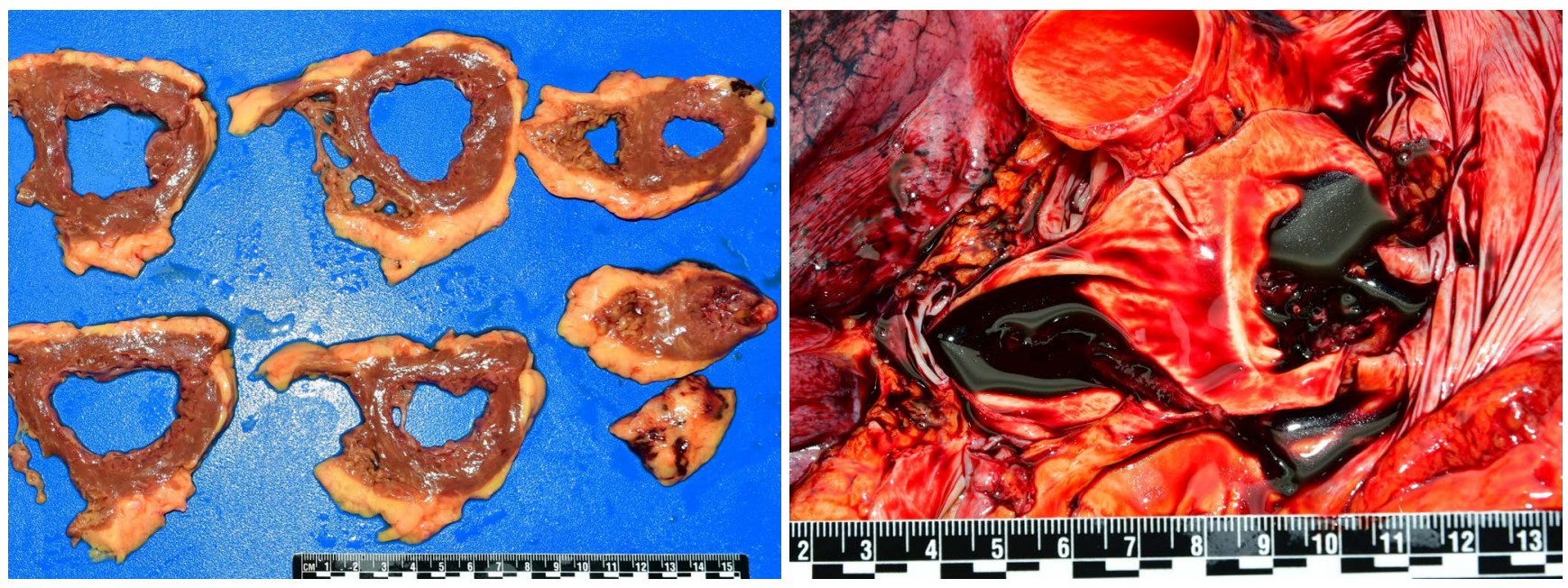

C

D

Fig. 1. Hemopericardium is observed in the pericardium (A), and epicardial hemorrhages are observed at the apex of the left ventricle (B). The myocardium and epicardium of the apex reveal multifocal hemorrhages related to focal rupture (C). Nonobstructive pulmonary thromboembolism are identified in the right and left pulmonary arteries (D). 

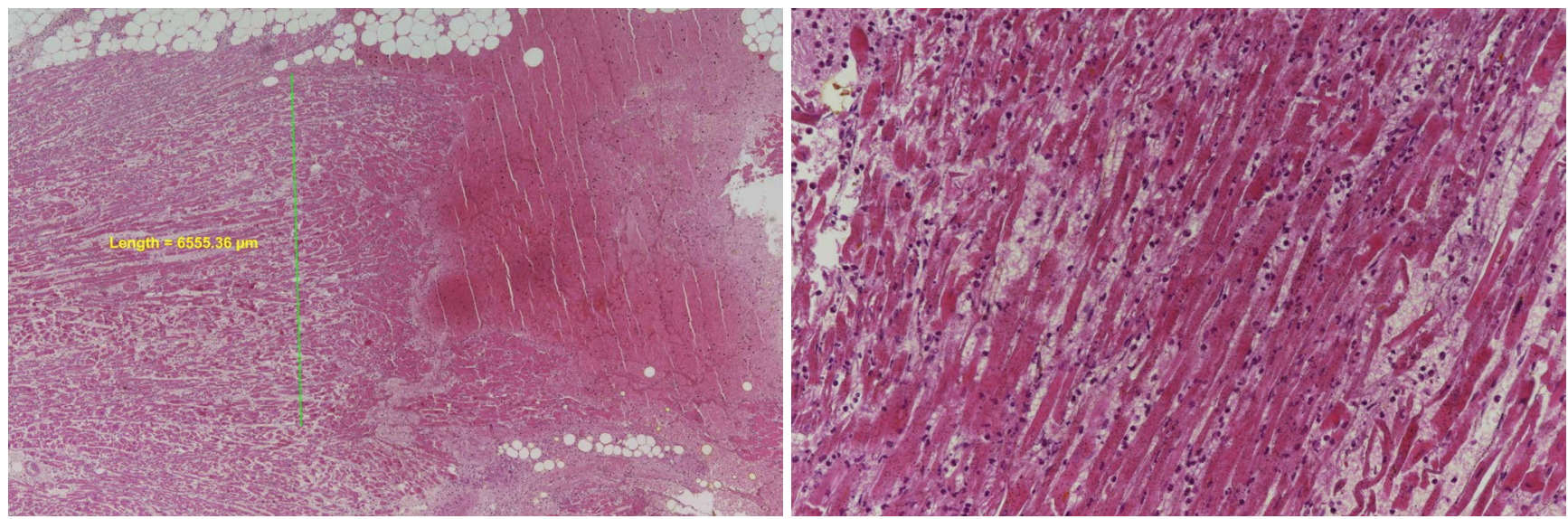

A

B
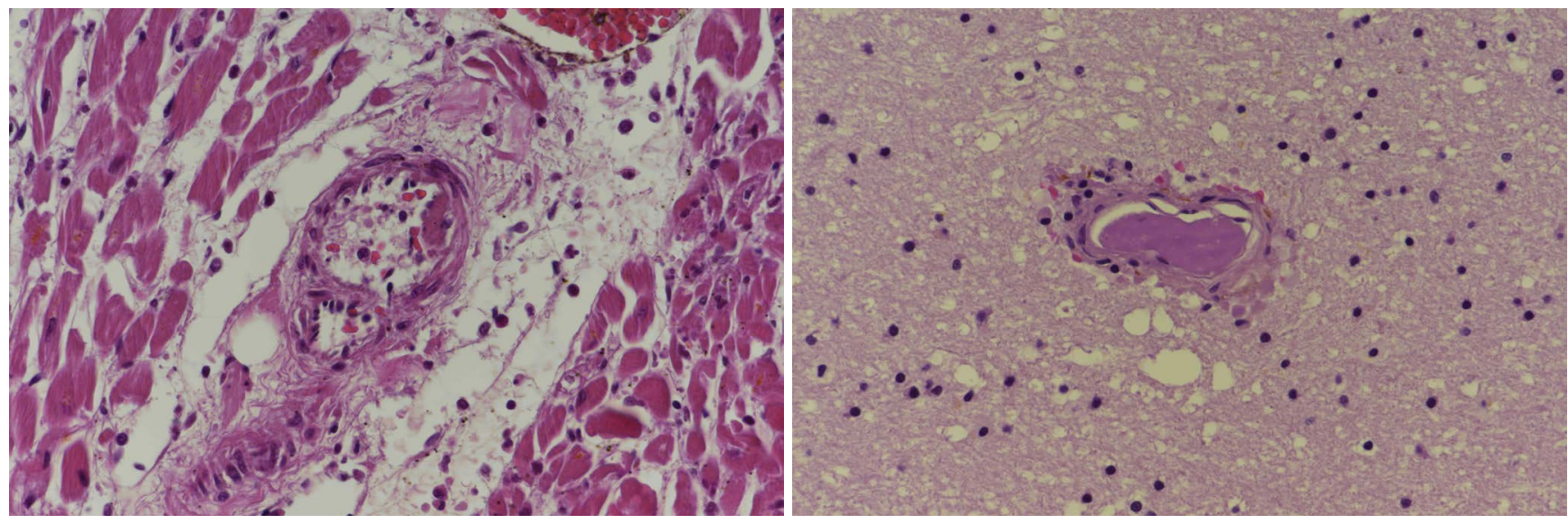

C

D
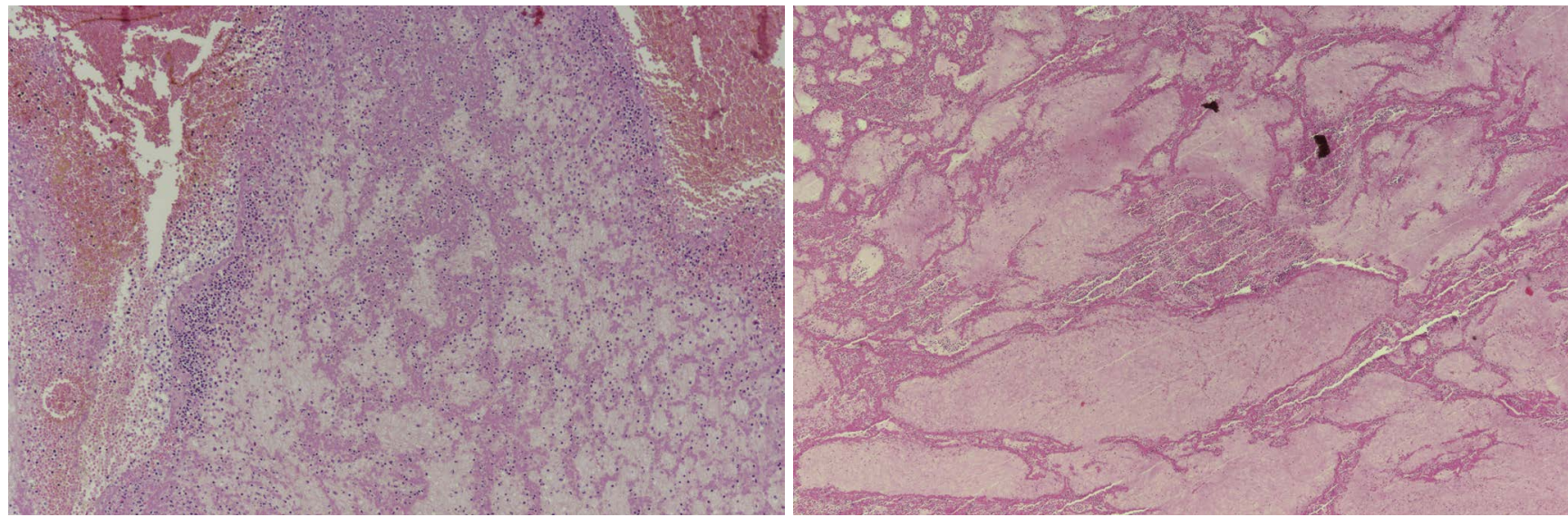

E

$\mathbf{F}$

Fig. 2. On microscopic examination, focal myocardial necrosis with rupture and hemorrhage is observed, and the length of the area is $<1 \mathrm{~cm}(\mathrm{H} \& \mathrm{E}$, $\times 40)(A)$. Myocardial necrosis with acute inflammation is also observed $(H \& E, \times 200)(B)$. Nonobstructive and eccentric fibrin microthrombi are observed in a small vessel in the myocardium $(\mathrm{H} \& \mathrm{E}, \times 400)(\mathbf{C})$. Fibrin microthrombi are also identified in a small vessel in the brain $(\mathrm{H} \& \mathrm{E}, \times 400)(\mathrm{D})$. Acute thrombi are observed in the pulmonary artery $(\mathrm{H} \& \mathrm{E}, \times 100)(\mathrm{E})$ and inferior vena cava $(\mathrm{H} \& \mathrm{E}, \times 40)(\mathrm{F})$. 
around focal myocardial necrosis. In patients with COVID-19, microthrombi could be explained by the previously known association between COVID-19 and hypercoagulability [5]. However, microthrombi in this case, could not be explained, because there were no culprit lesions, pathologic conditions, or past history, except for COVID-19 vaccination.

It is unclear whether the postmortem findings in this case could be considered as vaccine-induced immune thrombotic thrombocytopenia (VITT). Thrombotic events in the cerebral veins, deep veins of the leg, pulmonary arteries, and splanchnic vessels after COVID-19 vaccination (ChAdOx1 nCov-19) were reported as the clinical manifestations of VITT $[7,8]$. In this case, thrombosis (microthrombi in the small vessels in the myocardium and brain, nonobstructive thromboembolism in the pulmonary arteries and inferior vena cava) was also observed. However, the platelet count, D-dimer, and PF4 antibody couldn't be tested in order to confirm the diagnosis of VITT, because of postmortem changes and degeneration. To the best of our knowledge, there have been no reports or studies on whether COVID-19 vaccination is associated with myocardial injury caused by microthromobi, although the causal relationship between myocardial infarction and COVID-19 vaccination has been questioned [9]. Recently, another theory has suggested that vaccinationinduced free-floating Spike proteins may induce platelet aggregation, and interact with angiotensinconverting enzyme 2 receptor, leading to inflammation and thrombosis through disparity of pro-inflammation and anti-inflammation of the angiotensin system [10]. The pathologic condition of diabetes mellitus and hypertension including vascular dysfunction may be inclined to the proinflammatory arm of the reninangiotensin system induced by vaccination [11]. These possible pathogeneses may be helpful in explaining the overall findings of this case. Further comprehensive studies of additional cases are needed to establish the causal relationship between them.

Given that the deceased was a postmenopausal woman and the myocardial necrosis was observed in the apex of the heart, and ventricular thrombi was observed in other vasculature, Takotusbo cardiomyopathy (T-CMP) may be considered [12]. However, ventricular thrombus is a very rare complication of T-CMP, and venous thrombosis cannot be explained [12]. In addition, it is impossible to prove apical ballooning with postmortem findings. In this case, the deceased died suddenly and complained of only back pain one day prior to death. There were no significant symptoms, such as dyspnea or hypertension suggestive of severe left ventricular dysfunction.

This case revealed that postmortem examination is critical to confirm the cause of death in the cases of unexpected death after vaccination. Most major characteristic findings in this case could be identified only by postmortem examination. The clinical diagnosis of microthrombi in cardiac small vessels is difficult, and there is no laboratory method to diagnose it specifically. Clinical manifestations can be nonspecific and misleading. In patients with COVID-19, the sensitivity of the electrocardiogram approach to patients with myocardial injury was poor, and their clinical manifestations were variable and masked by respiratory symptoms [5]. In this case, it was difficult to anticipate any possibility of heart disease because the deceased complained of nonspecific symptoms such as pain at the vaccinated site, general weakness, and back pain.

In summary, we present a case of unusual myocardial infarction with microthrombi and thrombosis after COVID-19 vaccination (ChAdOx1 nCov-19), whose findings are similar to those of myocardial injury in patients with COVID-19, accompanied by thrombosis in other organs. Further studies are necessary to understand the pathogenesis and to clarify the causal relationship with COVID-19 vaccination. Given that the major unique features of this case were identified only by postmortem findings, meticulous postmortem examination is essential for unexpected death after vaccination.

ORCID: Sohyung Park: https://orcid.org/0000-00021987-7908; Byung-Ha Choi: https://orcid.org/00000002-9401-1725

Conflicts of Interest

No potential conflict of interest relevant to this article was reported. 


\section{Acknowledgments}

This study was approved by the National Forensic Service Institutional Review Board (906-210914-BR001-01).

\section{References}

1. Jeon M, Kim J, Oh CE, et al. Adverse events following immunization associated with the first and second doses of the ChAd0x1 nCoV-19 vaccine among healthcare workers in Korea. Vaccines (Basel) 2021;9:1096.

2. Falk E. Unstable angina with fatal outcome: dynamic coronary thrombosis leading to infarction and/or sudden death: autopsy evidence of recurrent mural thrombosis with peripheral embolization culminating in total vascular occlusion. Circulation 1985;71:699-708.

3. Davies MJ, Thomas AC, Knapman PA, et al. Intramyocardial platelet aggregation in patients with unstable angina suffering sudden ischemic cardiac death. Circulation 1986;73:418-27.

4. Schwartz RS, Burke A, Farb A, et al. Microemboli and microvascular obstruction in acute coronary thrombosis and sudden coronary death: relation to epicardial plaque histopathology. J Am Coll Cardiol 2009;54:2167-73.
5. Pellegrini D, Kawakami R, Guagliumi G, et al. Microthrombi as a major cause of cardiac injury in COVID-19: a pathologic study. Circulation 2021;143:1031-42.

6. Bois MC, Boire NA, Layman AJ, et al. COVID-19-associated nonocclusive fibrin microthrombi in the heart. Circulation 2021;143:230-43.

7. Greinacher A, Thiele T, Warkentin TE, et al. Thrombotic thrombocytopenia after ChAdOx1 nCov-19 vaccination. $\mathrm{N}$ Engl J Med 2021;384:2092-101.

8. Pavord S, Scully M, Hunt BJ, et al. Clinical features of vaccineinduced immune thrombocytopenia and thrombosis. N Engl J Med 2021;385:1680-9.

9. Chatterjee S, Ojha UK, Vardhan B, et al. Myocardial infarction after COVID-19 vaccination-casual or causal? Diabetes Metab Syndr 2021;15:1055-6.

10. Angeli F, Spanevello A, Reboldi G, et al. SARS-CoV-2 vaccines: lights and shadows. Eur J Intern Med 2021;88:1-8.

11. Gheblawi M, Wang K, Viveiros A, et al. Angiotensin-converting enzyme 2: SARS-CoV-2 receptor and regulator of the reninangiotensin system: celebrating the 20th Anniversary of the Discovery of ACE2. Circ Res 2020;126:1456-74.

12. Templin C, Ghadri JR, Diekmann J, et al. Clinical features and outcomes of Takotsubo (stress) cardiomyopathy. N Engl J Med 2015;373:929-38. 